\title{
Die deutsche Interpretation italienischer Schichttechnik
}

Style Italiano - das ist das Prinzip italienischer Leichtigkeit übertragen auf die Komposit-Füllungstherapie. Das Konzept von den Italienern Prof. Dr. Angelo Putignano und Dr. Walter Devoto basiert auf einer Rezeptur und wenigen ausgewählten Zutaten. Damit lassen sich auf einfache Weise ästhetische Frontzahnrestaurationen herstellen. Die dafür erforderlichen Materialien und das entsprechende Know-how werden von 3M ESPE im Rahmen von regelmäßig organisierten Fortbildungsveranstaltungen vermittelt. Die Gelegenheit nutzten Anfang April 2014 rund 20 Zahnärzte, die in Heusenstamm an dem vierstündigen Event mit dem Titel „Style Italiano
- Rezepte für ein strahlendes Lächeln“ teilnahmen.

$\mathrm{Zu}$ Beginn wird mit dem bevorzugten Füllungsmaterial - Filtek Supreme XTE Universal Komposit von 3M ESPE - ein individueller Farbschlüssel erstellt. Dieser ermöglicht es, bei der Farbbestimmung bereits die spezifischen optischen Eigenschaften des Materials zu berücksichtigen und somit vorhersagbare Ergebnisse zu produzieren. Das Wichtigste dabei: Für jede hochästhetische Frontzahnrestauration werden nur 2 unterschiedliche Opazitäten benötigt. Für eine natürliche Ästhetik sorgt die vestibuläre Schmelzschicht, deren Stärke exakt $0,5 \mathrm{~mm}$ beträgt. Im anschließenden praktischen Teil

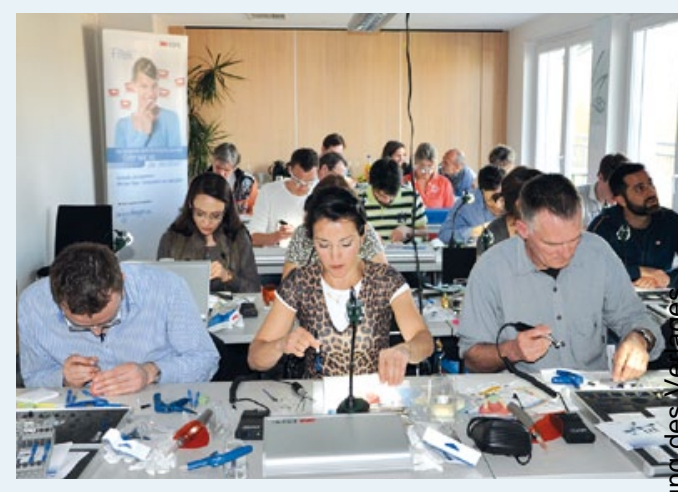

wurde die Schichttechnik demonstriert und von den Teilnehmern umgesetzt.

Nach einer Pressemitteilung der

3M Deutschland GmbH, Seefeld

\section{Chirurgie}

\section{Verlåsslich in der Funktion, einfach in der Handhabung}

Nobel Biocare erweitert sein Produktportfolio nun mit Creos xenoprotect um eine bioresorbierbare nicht vernetzte Kollagenmembran auf porciner Basis. Die Membran eignet sich für Verfahren der gesteuerten Knochen- (GBR) sowie Geweberegeneration (GTR) und hat entscheidende Vorteile: Sie schafft ein für die Knochenregeneration günstiges Umfeld im Defektareal, weil die Migration unerwünschter Zellen aus dem umgebenden Weichgewebe verhindert und zugleich das Wachstum osteogener Zellen unterstützt wird. Und: Das operative Handling mit Creos xenoprotect ist einfach, weil die
Membran beidseitig verwendet werden kann und sich dank ihrer hohen Festigkeit gut in Form bringen lässt, um Knochendefekte sicher abzudecken. Die natürliche Barrieremembran besteht aus einem starken Geflecht hochgereinigter Schweinekollagenfasern, die mit ebenso hochreinen Schweineelastinfasern vermischt sind. Dadurch ist die Membran sehr fest und stabil - wichtige Eigenschaften, um die Migration von Augmentationsmaterial zu verhindern und um eine möglichst langsame Biodegradation der Membran zu erreichen. Denn bei resorbierbaren Kollagenmembranen kommt es für den
Therapierfolg gerade darauf an, dass die Membran trotz ihres biologischen Abbaus ausreichend stabil bleibt. Hier zeigt Creos xenoprotect eine deutlich verlängerte Barrierefunktion trotz des Revaskulierungsverhaltens und der Gewebekompatibilität. Die Membran integriert sich zunächst in das umgebende Gewebe, um sich dann abzubauen. Die Membran ist ab sofort in 3 verschiedenen Größen erhältlich $(10 \times 15 \mathrm{~mm} ; 25 \times 30 \mathrm{~mm}$ und $30 \times 40 \mathrm{~mm}$ ).

Nach einer Pressemitteilung der Nobel Biocare Deutschland GmbH, Köln Internet: www.nobelbiocare.com

\section{Prophylaxe}

Die neue Prosonic micro 2, aus dem miradent Prophylaxesortiment, ist eine batteriebetriebene Schallzahnbürste. Mit 30000 Vibrationen pro Minute reinigt sie effizienter die Zähne als herkömmliche Maßnahmen. Aufgrund der Mikrovibrationen werden Bakterien, Plaque und Verfärbungen optimal entfernt. Der integrierte Zungenreiniger auf der Bürstenkopfrückseite dient zur einfachen Entfernung von Zungenbelägen. Ein kleiner Abstandshalter auf der Rückseite ermög- licht ein hygienisches Ablegen der Zahnbürste. Die Bürste passt in jedes Reisegepäck und eignet sich ideal für unterwegs. Der austauschbare Bürstenkopf hält ca. 2-3 Monate. Das Ersatzbürstenset besteht aus 2 Büstenköpfen +4 farbcodierten Ringen. Die neue miradent Prosonic micro 2 ist ab sofort im Fachhandel erhältlich.

Nach einer Pressemitteilung der Hager \& Werken GmbH \& Co. KG, Duisburg Internet: www.hagerwerken.de, www.miradent.de

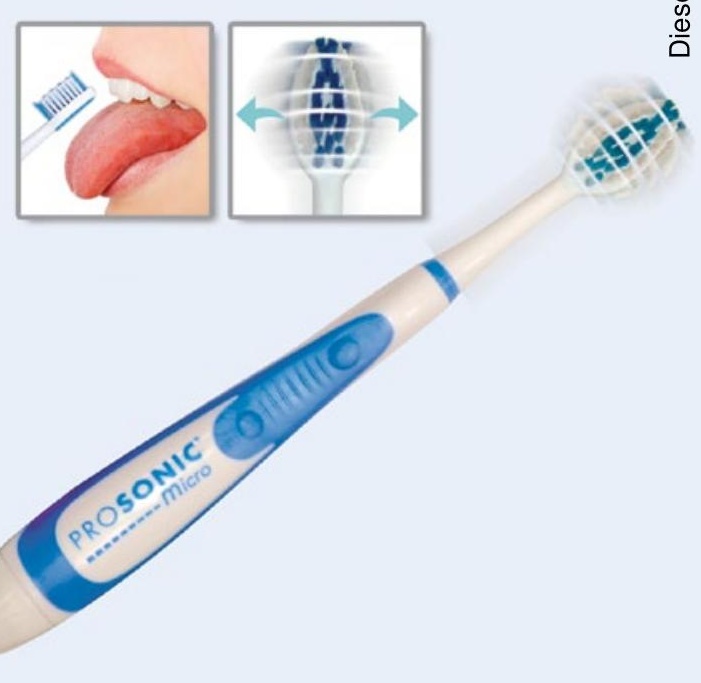


Einfach Fan-tastisch

\section{Exklusive Pakete -}

\section{jetzt zum WM-Sonderpreis}

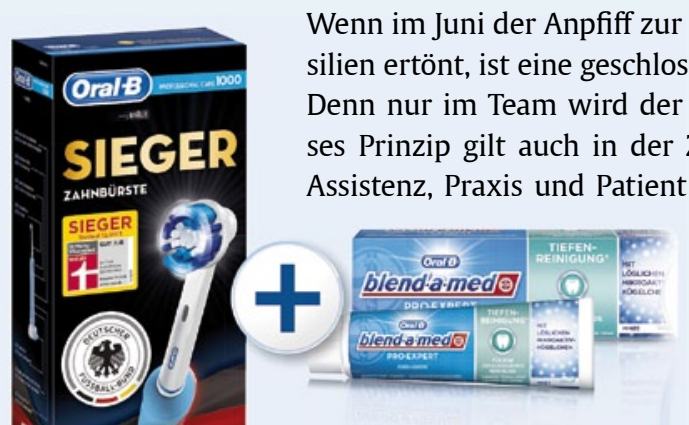

Wenn im Juni der Anpfiff zur Fußball-Weltmeisterschaft in Brasilien ertönt, ist eine geschlossene Mannschaftsleistung gefragt. wird der Traum vom Erfolg Realität - dieses Prinzip gilt auch in der Zahnheilkunde. Ob Zahnarzt und Assistenz, Praxis und Patient oder elektrische Zahnbürste und Zahncreme, nur gemeinsam lässt sich das Ziel „Verbesserung der Mundgesundheit" erreichen. Aus diesem Grund bietet Oral-B zur Fußball-WM exklusive Praxis-Pakete für das Prophylaxeteam zum Sonderpreis: Jedes beinhaltet eine offizielle Fan-Zahnbürste des DFB, die Oral-B Professional Care 1000, und 12 Tuben der OralB/ blendamed PRO-EXPERT-Tiefenreinigung.

Noch bis zum 30. Juni 2014 lässt sich das Dreamteam zum exklusiven Paketpreis für Zahnarztpraxen in die Startaufstellung berufen. Bestellen geht ganz einfach: entweder per Faxantwort auf das Mailing im Briefkasten, beim zuständigen Oral-B-Dentalfachberater oder direkt online auf www.dentalcare.com.

Nach einer Pressemitteilung der

Procter \& Gamble Germany GmbH, Schwalbach am Taunus

Kompositrestaurationen auf dem YouTube-Kanal

\section{Schritt für Schritt mit Tipps und Tricks}

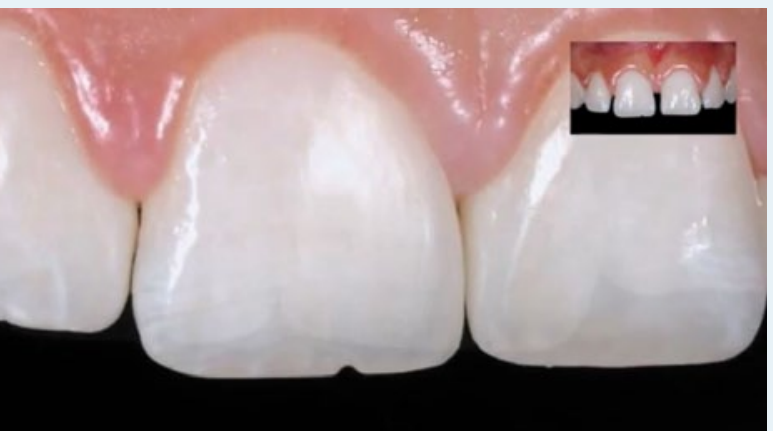

Diastemaschluss durch eine minimalinvasive direkte Kompositrestauration

Wie überzeugend Frontzahnversorgungen mit einem ästhetischen Ergebnis gelingen können, zeigen jetzt 2 kurze Filme, die Heraeus Kulzer Zahnärzten über seinen eigenen Kanal auf YouTube kostenlos zur Verfügung stellt. Darin demonstriert Ulf Krueger-Janson, Zahnarzt aus Frankfurt am Main und Vizepräsident der DGR 2 Z, sein Vorgehen an 2 klinischen Fällen und verrät dabei Tipps und Tricks zu den einzelnen Schritten. Krueger-Janson dokumentiert in seiner Praxis einen Diastemaschluss sowie eine Klasse-IV-Restauration an Zahn 11. Der erfahrene Behandler schildert die Herausforderungen der jeweiligen Ausgangssituation und hebt im Behandlungsverlauf einzelne Schritte besonders hervor. Mit den beiden ästhetischen Ergebnissen zeigen die Komposite Venus Diamond und Venus Pearl erneut, dass sie mehr als nur Standardindikationen abdecken, sondern auch für anspruchsvolle Fälle zuverlässige Lösungen bieten. Zu sehen sind die beiden Videos - neben weiteren interessanten Versorgungssituationen - über den Heraeus Kulzer-Kanal. Wer sich zudem dafür registriert, wird regelmäßig über Neuigkeiten informiert. Die Videos sind durch die Suchfunktion oder direkt über den QR-Code zu erreichen.

Nach einer Pressemitteilung der

Heraeus Kulzer GmbH, Hanau

Internet: www.heraeus-kulzer.com
Neues Kundenmagazin

\section{Digitale Ausgabe als App} oder Web-Version bietet Zusatzinformationen

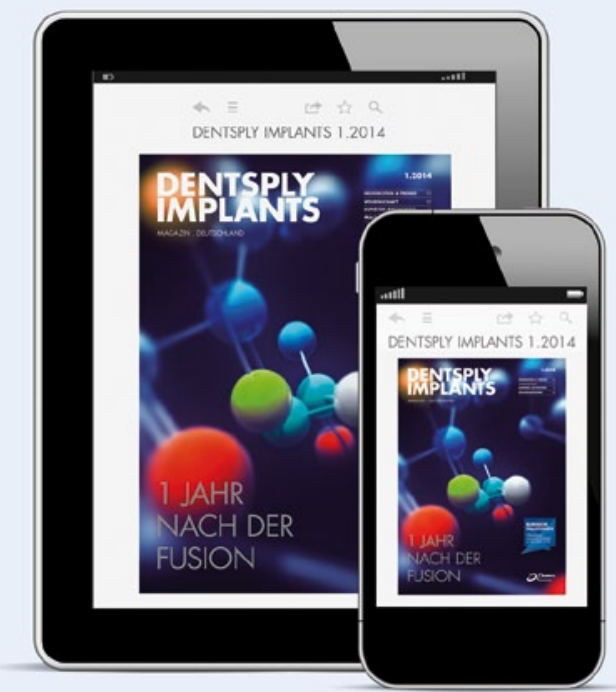

Mit einer Auflage von 25000 Exemplaren startet das neue Kundenmagazin von DENTSPLY Implants auf dem deutschen Markt. Das DENTSPLY Implants-Magazin verbindet die beliebtesten Elemente der bisherigen Kundenzeitschriften iDENTity (DENTSPLY Friadent) und InSight (Astra Tech).

Im Mittelpunkt stehen Fallberichte, die dem Anwender zahlreiche Anregungen bieten - von patientenfreundlichen implantologischen Konzepten über zuverlässige augmentative Verfahren bis hin zu präzisesten prothetischen Lösungen. Neben Informationen über Produktneuheiten, Fortbildungen und Veranstaltungen, geben Unternehmensvertreter auch Einblicke in den Kundenservice und in den Bereich der wissenschaftlichen Dokumentationen. Aber auch aktuelle Trends wie Frauen in der Zahnmedizin oder die digitale Zukunft der Zahnmedizin werden im neuen Magazin thematisiert. Die Leser können Fragen stellen, die von Experten beantwortet und in der nächsten Ausgabe veröffentlicht werden. Neben der Printausgabe ist das DENTSPLY Implants-Magazin Deutschland auch digital verfügbar: In der Web- sowie in der App-Version für iPhone und iPad (ab Ende Mai auch für Android) gibt es Zusatzinhalte wie Bildergalerien, Videos und Links zu weiterführenden Informationen. Das neue Kundenmagazin sowie alle weiteren Informationen und Links zu den digitalen Ausgaben stehen unter www.dentsplyimplants-magazin.de zur Verfügung. Die 2. Ausgabe erscheint im Herbst 2014.

Nach einer Pressemitteilung der Dentsply Implants GmbH, Mannheim 
30. Geburtstag

\section{Jubiläum mit attraktiven} Angebotspreisen

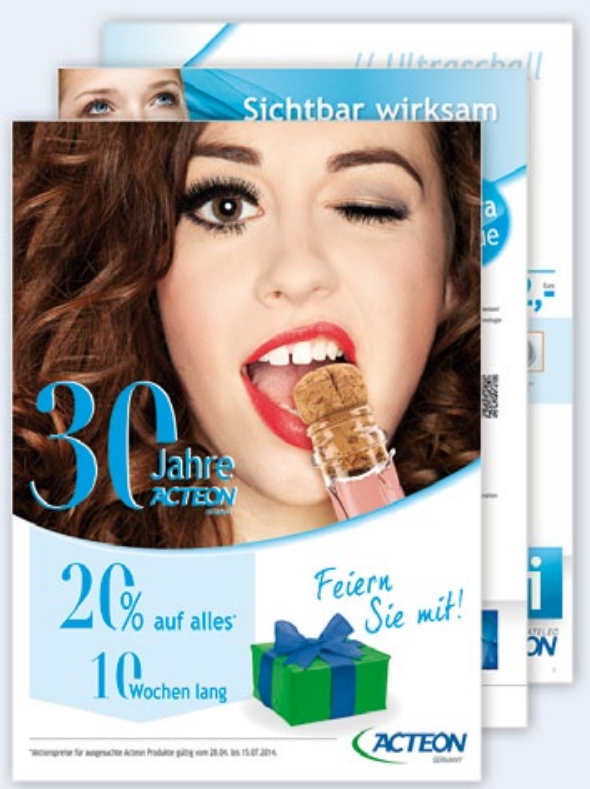

Dieses Jahr feiert die ACTEON Germany GmbH ihren 30. Geburtstag - Anlass genug, eine ganz besondere Aktion für die Kunden zu starten. Ab dem 28. April gibt es 10 Wochen lang 20\% Rabatt auf ausgewählte Geräte und alle Instrumentenkits. In den Bereichen Prophylaxe, Chirurgie und Implantologie, Polymerisation, intraorale Bildgebung und Röntgen wird für jeden Spezialisten das passende Gerät angeboten. Dabei beinhaltet jedes Produktsegment neben einer Standardvariante auch eine High-End-Lösung mit zusätzlichen Funktionalitäten: Ultraschallgeneratoren mit Plaque-Erkennung (Newtron P5 XS B.LED) oder ohne (P5 Newtron XS); Pulverstrahlgeräte als Tischvariante (Air Max 2) oder Handy (Air-NGo); Ultraschallgeräte für die Knochenchirurgie mit eingebautem Chirurgiemotor (ImplantCenter 2 LED) oder als Einzellösung (Piezotome 2); Intraoralkameras mit zusätzlicher Plaque- und Karieserkennung (SoproCare) oder in der Standardversion (Sopro 617) sowie HochfrequenzRöntgengeräte mit intelligenter Strahlenbegrenzung (X-Mind unity und Sensor Sopix Inside) oder in der kostengünstigeren Variante (X-Mind DC). Neben hochqualitativen Investitionsgütern sind auch Verbrauchsmaterialien wie PolishingPulver sowie alle Ultraschall- und Chirurgie-Instrumentenkits im Angebotsportfolio enthalten. Der Aktionszeitraum für das Jubiläumsangebote läuft vom 28. April bis zum 15. Juli 2014. Der Flyer kann auf der Firmenwebsite unter http:// de.acteongroup.com/ heruntergeladen werden.

Nach einer Pressemitteilung der Acteon Germany GmbH, Mettmann

Internet: www.de.acteongroup.com
Patientenwunsch

\section{Differenzierte Be- und Enttäubung für einen angenehmeren Zahnarztbesuch}

An einem Zahnarztbesuch stört Patienten am häufigsten das Taubheitsgefühl in Lippen und Zunge nach der Behandlung mit einer dentalen Lokalanästhesie mit Vasokonstriktor. Jeder 2. Patient empfindet dieses Gefühl als unangenehm. 23\% der Befragten, die auf eine örtliche Betäubung verzichten, begründen dies mit der störenden Gefühllosigkeit der Weichgewebe. Zu diesen Ergebnissen kommt eine repräsentative Umfrage des Marktforschungsinstituts GfK, welche die Bevölkerung in Deutschland ab 14 Jahren nach ihren Zahnarzterfahrungen befragte. Demnach würde für

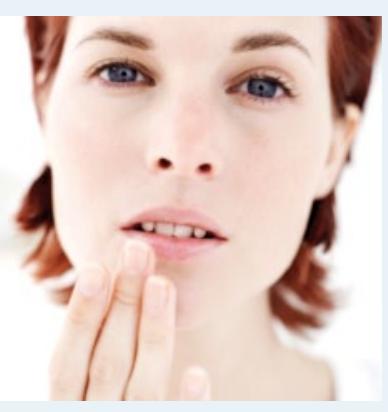
$56,8 \%$ ein Zahnarztbesuch angenehmer werden, wenn eine moderne zahnärztliche Betäubung nach der Behandlung das Taubheitsgefühl auch wieder aufhebt. Diese Möglichkeit haben Zahnärzte mit dem Präparat OraVerse ${ }^{\circledR}$. Mit dieser zusätzlichen Phentolaminmesilat-Injektion bieten Zahnärzte ihren Patienten nach nicht chirurgischen Routineeingriffen wie Präparationen von Füllungskavitäten und Inlays, Kronen und Brückenpfeilern eine individuelle Gesundheitsleistung. Durch die Enttäubung verkürzt sich die Dauer des Taubheitsgefühls um mehr als $50 \%$. Damit Zahnärzte ihren Patienten die Vorteile der Enttäubung erläutern können, stellt Sanofi Patientenbroschüren und Plakate zur Verfügung. Diese sind für Zahnärzte per E-Mail an medinfo.de@sanofi-aventis.com bestellbar.

Nach einer Pressemitteilung der

Sanofi-Aventis Deutschland GmbH, Frankfurt

Internet: www.sanofi.de

\section{Neues Innovationsportal}

\section{Kunden als produktive Ideengeber}

Ab sofort besteht bei Dürr Dental die Möglichkeit, Ideen schnell und unkompliziert beim Unternehmen einzubringen. Immer wieder kommen Kunden und Tüftler auf Dürr Dental zu, um neue Ideen zu diskutieren. Viele gute Ideen zu Optimierungen entstehen aber auch in den Praxen, in denen mit den Dürr Dental Produkten täglich gearbeitet wird. Aus Sicht der Entwicklungsleitung des Unternehmens hat die Vergangenheit gezeigt, dass beim täglichen praxisnahen Umgang mit den Produkten immer wieder interessante Ansätze für Innovationen entstehen, welche sich in Neuentwicklungen widerspiegeln. Um für Interessierte diesen Schritt einfacher zu gestalten, hat das Unternehmen unter www.

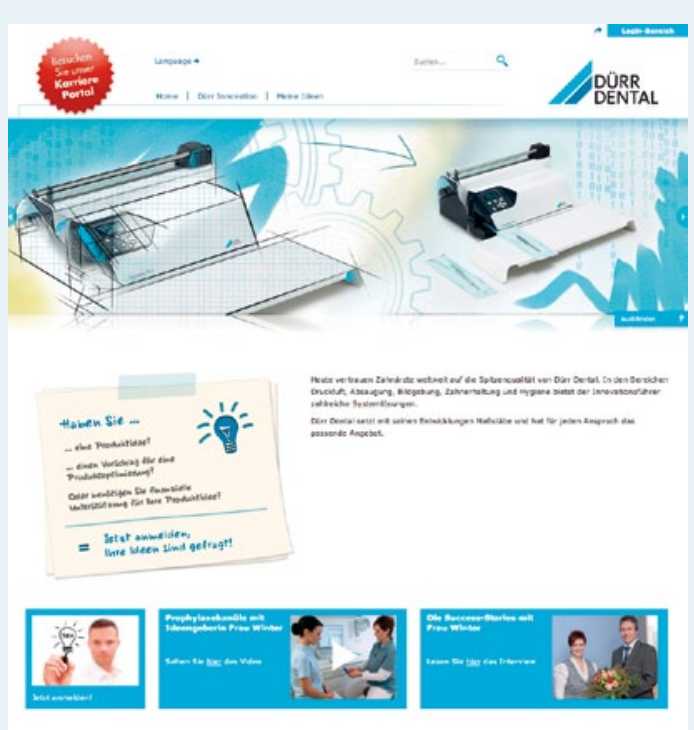

duerr-innovation.de ein neues Innovationsportal eingerichtet.

Nach einer Pressemitteilung der Dürr Dental AG, Bietigheim-Bissingen

Internet: www.duerrdental.com 
